\title{
Branched-chain amino acid levels are associated with improvement in insulin resistance with weight loss
}

\author{
S. H. Shah • D. R. Crosslin • C. S. Haynes - S. Nelson - C. B. Turer • R. D. Stevens • \\ M. J. Muehlbauer • B. R. Wenner • J. R. Bain • B. Laferrère • P. Gorroochurn • \\ J. Teixeira • P. J. Brantley • V. J. Stevens • J. F. Hollis • L. J. Appel • L. F. Lien • \\ B. Batch • C. B. Newgard $\cdot$ L. P. Svetkey
}

Received: 18 August 2011 / Accepted: 28 September 2011 /Published online: 8 November 2011

(C) Springer-Verlag 2011

\begin{abstract}
Aims/hypothesis Insulin resistance (IR) improves with weight loss, but this response is heterogeneous. We hypothesised that metabolomic profiling would identify biomarkers predicting changes in IR with weight loss.

Methods Targeted mass spectrometry-based profiling of 60 metabolites, plus biochemical assays of NEFA, $\beta$ hydroxybutyrate, ketones, insulin and glucose were performed in baseline and 6 month plasma samples from 500 participants who had lost $\geq 4 \mathrm{~kg}$ during Phase I of the Weight Loss Maintenance (WLM) trial. Homeostatic model assessment of insulin resistance (HOMA-IR) and change in HOMA-IR with weight loss ( $\triangle$ HOMA-IR) were calculated. Principal components analysis (PCA) and mixed models adjusted for race, sex, baseline weight, and amount of
\end{abstract}

Electronic supplementary material The online version of this article (doi:10.1007/s00125-011-2356-5) contains peer-reviewed but unedited supplementary material, which is available to authorised users.

\section{S. H. Shah $(\square)$ \\ Department of Medicine, DUMC, \\ Duke University Medical Center, \\ Box 3445, Durham, NC 27710, USA \\ e-mail:svati.shah@duke.edu}

S. H. Shah • L. F. Lien • B. Batch • C. B. Newgard - L. P. Svetkey Department of Medicine, Duke University Medical Center,

Durham, NC, USA

S. H. Shah $\cdot$ C. S. Haynes

Center for Human Genetics, Duke University Medical Center,

Durham, NC, USA

S. H. Shah • R. D. Stevens - M. J. Muehlbauer - B. R. Wenner •

J. R. Bain · C. B. Newgard - L. P. Svetkey

Sarah W. Stedman Nutrition and Metabolism Center,

Duke University,

Durham, NC, USA weight loss were used; findings were validated in an independent cohort of patients $(n=22)$.

Results Mean weight loss was $8.67 \pm 4.28 \mathrm{~kg}$; mean $\triangle$ HOMAIR was $-0.80 \pm 1.73$, range -28.9 to 4.82 ). Baseline PCAderived factor 3 (branched chain amino acids [BCAAs] and associated catabolites) correlated with baseline HOMA-IR ( $r=$ $0.50, p<0.0001)$ and independently associated with $\triangle$ HOMA-IR $(p<0.0001)$. $\triangle$ HOMA-IR increased in a linear fashion with increasing baseline factor 3 quartiles. Amount of weight loss was only modestly correlated with $\triangle$ HOMA-IR $(r=0.24)$. These findings were validated in the independent cohort, with a factor composed of BCAAs and related metabolites predicting $\triangle$ HOMA-IR ( $p=0.007$ ).

Conclusions/interpretation A cluster of metabolites comprising BCAAs and related analytes predicts improvement
B. Laferrère

New York Obesity Nutrition Research Center,

St. Luke's Roosevelt Hospital Center,

New York, NY, USA

P. Gorroochurn

Department of Biostatistics, Columbia University

College of Physicians and Surgeons,

New York, NY, USA

J. Teixeira

Division of Minimally Invasive Surgery,

St. Luke's Roosevelt Hospital Center,

New York, NY, USA

B. Laferrère $\cdot J$. Teixeira

Columbia University College of Physicians and Surgeons,

New York, NY, USA 
in HOMA-IR independent of the amount of weight lost. These results may help identify individuals most likely to benefit from moderate weight loss and elucidate novel mechanisms of IR in obesity.

Keywords Amino acids · Biomarker - Insulin resistance . Metabolites $\cdot$ Weight loss

$\begin{array}{ll}\text { Abbreviations } \\ \text { BCAA } & \text { Branched-chain amino acids } \\ \text { HEI } & \text { Healthy Eating Index } \\ \text { IR } & \text { Insulin resistance } \\ \text { IRB } & \text { Institutional Review Board } \\ \text { PCA } & \text { Principal components analysis } \\ \text { WLM trial } & \text { Weight Loss Maintenance trial }\end{array}$

Insulin resistance (IR) is a core pathophysiological mechanism that develops in concert with beta cell failure to cause type 2 diabetes mellitus, a disease with enormous public health impact. Over 23 million individuals in the US and 285 million individuals globally are afflicted with this condition (International Diabetes Federation). IR and type 2 diabetes mellitus are strongly associated with increasing body weight. Indeed, the adverse health consequences of overweight/ obesity occur primarily as a result of effects on cardiovascular disease risk factors including type 2 diabetes mellitus and IR. Fortunately, these effects are reversible with weight loss in a graded fashion. In fact, one does not need to achieve a normal BMI to reap health benefits from weight loss. For example, a mean weight loss of only $5.5 \mathrm{~kg}$ in overweight and obese

\section{P. J. Brantley}

Pennington Biomedical Research Center,

Baton Rouge, LA, USA

V. J. Stevens · J. F. Hollis

Health Science Programs, Center for Health Research,

Kaiser Permanente Northwest,

Portland, OR, USA

\section{J. Appel}

Welch Center for Prevention, Epidemiology and Clinical Research, Johns Hopkins University School of Medicine, Baltimore, MD, USA

\section{B. Newgard}

Department of Pharmacology and Cancer Biology,

Duke University,

Durham, NC, USA

C. B. Turer

Department of Medicine, UT Southwestern Medical Center, Dallas, TX, USA

D. R. Crosslin $\cdot$ S. Nelson

Department of Biostatistics, University of Washington,

Seattle, WA, USA adults with insulin resistance reduced the incidence of type 2 diabetes mellitus by $58 \%$ [1]. However, the extent to which weight loss improves IR appears to be heterogeneous [2], an observation that has not been fully explained. Molecular profiling tools may help dissect this heterogeneity, thereby identifying individuals most likely to have health benefits from weight loss, increasing our understanding of pathophysiology and potentially identifying new targets for prevention and treatment.

The Weight Loss Maintenance (WLM) Study was a randomised clinical trial that included an intensive behavioural weight loss phase followed by randomisation to one of three maintenance strategies [3]. We evaluated the heterogeneity of improvement in IR with weight loss in the WLM study. We hypothesised that metabolomic profiling would identify novel biomarkers that predict changes in IR with weight loss.

\section{Methods}

\section{Study population}

WLM clinical trial The WLM study was a multi-centre, randomised trial (clinicaltrials.gov NCT00054925) to determine the effects of two weight loss maintenance interventions compared with a usual care control group [3]. Eligibility requirements included a BMI consistent with overweight or obesity $\left(25-45 \mathrm{~kg} / \mathrm{m}^{2}\right)$, taking medication for hypertension and/or dyslipidaemia, and age 25 years or older. The criteria for medications for hypertension and/or dyslipidaemia was mandated by the WLM trial, to enrol patients with more established cardiovascular risk factors that could be potentially modified by weight loss. Exclusion criteria included medication-treated type 2 diabetes mellitus. In phase I of the trial, a group-based behavioural intervention was administered over 6 months to 1,685 participants enrolled in the trial (Electronic supplementary material [ESM] Fig. 1) [3]. At entry into phase I and at each subsequent visit, blood samples were collected from all participants via peripheral vein phlebotomy, immediately processed to separate serum and plasma, and frozen at $-70^{\circ} \mathrm{C}$ within $1-2 \mathrm{~h}$. Participants were asked to fast for $8-14 \mathrm{~h}$; if they reported fasting for fewer than 8 or greater than $14 \mathrm{~h}$, the visit was rescheduled. Participants able to lose at least $4 \mathrm{~kg}$ of weight during phase I were randomised in phase II; 1,032 study participants met this requirement. Detailed methods for variables collected in the WLM trial have been published previously [3]. Dietary intake was measured by the Block Food Frequency Questionnaire [4], from which the Healthy Eating Index (HEI) was calculated [5].

WLM subset used for this study From participants who were randomised in phase II $(n=1,032)$, we randomly 
selected 500 participants who had blood samples available at all time points. On this subset, metabolomic profiling was performed at baseline and 6 month plasma samples, i.e. before and after phase I weight loss (ESM Fig. 1).

The current analysis was approved by both the Steering Committee of the WLM Collaborative Research Group and the Duke Institutional Review Board (IRB). Use of stored WLM data and samples was approved by the IRB of each WLM clinical site and the coordinating centre.

Validation cohort Significant findings in the WLM study were tested in an independent cohort of patients undergoing weight loss intervention at the New York Obesity Research Center. In this cohort, morbidly obese patients with type 2 diabetes mellitus were studied before and 1 month after gastric bypass surgery $(n=12)$ and before and after an equivalent level of weight loss in response to a low calorie diet $(n=10)$. Detailed methods have been described [6]. Fasting blood samples were obtained before each weight loss intervention and 1 month after gastric bypass surgery, and for the diet group, after the patients had lost approximately $10 \mathrm{~kg}$, to match the weight loss observed in the gastric bypass group. Two-thirds of this cohort was on medication for diabetes prior to weight loss.

\section{Laboratory analysis}

We measured 45 acylcarnitines and 15 amino acids by tandem mass spectrometry (ESM Tables 1 and 2) in plasma samples, using stable isotope dilution for quantification, as previously described [7-9]. Ketones, $\beta$-hydroxybutyrate, total NEFA, glucose and insulin were measured by standard methods. Sample preparation methodology and coefficients of variation have been reported [7,9]. The laboratory (Sarah W. Stedman Nutrition and Metabolism Center Metabolomics/Biomarker Core Laboratory) was blinded to weight loss status and all samples from both cohorts were run in two complete batches.

\section{Statistical analysis}

IR was measured by the HOMA-IR index calculation as we have done previously [10]. Baseline HOMA, 6 month HOMA and change in HOMA-IR ( $\triangle$ HOMA-IR, baseline minus 6 months) were calculated, with $\triangle$ HOMA-IR as the primary outcome of interest. Mixed models with fixed effects were used to identify baseline clinical variables associated with $\triangle \mathrm{HOMA}-\mathrm{IR}$, to be used as covariables for adjustment in subsequent models inclusive of metabolomic biomarkers.

Principal components analysis (PCA) was used to reduce the large number of correlated metabolites into clusters of fewer uncorrelated factors, as has been done previously [7, 9]. Metabolites with $>25 \%$ ' 0 ' values (i.e. below the lower limits of quantification for that assay) were not analysed further (two acylcarnitine species). PCA was performed on baseline levels of acylcarnitines, amino acids, NEFA, ketones and $\beta$-hydroxybutyrate. Factors with eigenvalues $\geq 1.0$ were retained and varimax rotation performed to produce interpretable factors. Metabolites having a factor load (the weight by which each standardized original metabolite was multiplied to get the component score) with an absolute value $\geq 0.4$ were reported as composing a given factor. Scoring coefficients were used to calculate baseline metabolomic factor scores for each individual (weighted sum of the values of the standardised metabolites within that factor, weighted on the factor loading for each individual metabolite). Spearman correlation coefficients were used to assess the correlation between HOMA-IR, $\triangle$ HOMA-IR and metabolomic factors. To better understand the direction of effect of the relationship between metabolomic factors and $\triangle$ HOMA-IR, significant factors were divided into quartiles with equal numbers of individuals (i.e. $n=125$ in each quartile). Univariate and multivariable mixed models were used to assess the relationship between baseline metabolomic factors, HOMA-IR and $\triangle$ HOMA-IR. Paired $t$ tests were used to assess significance of change in metabolite levels with weight loss.

As all analyses were exploratory in nature and given the co-linearity of the metabolites, two-sided $p$ values unadjusted for multiple comparisons are presented; results interpreted in the context of a conservative Bonferroni correction are also reported. Nominal statistical significance was defined as $p \leq 0.05$. Statistical analyses were performed using SAS version 9.1 (SAS Institute, Cary NC).

\section{Results}

Table 1 presents baseline characteristics of the 1,032 WLM participants who lost at least $4 \mathrm{~kg}$ of weight in phase I and for the subset of 500 participants included in this study. As expected given inclusion criteria, there was a high prevalence of use of medication for hypertension and dyslipidaemia, and all participants were on at least one form of such medication. The distribution of clinical variables was similar across both groups, reflecting random sampling. In the subset of 500 WLM participants detailed in this study, the mean weight loss was $8.67 \pm 4.28 \mathrm{~kg}$ and mean $\triangle$ HOMA was $-0.80 \pm 1.73$, with a range of -28.9 to 4.82. Although there was modest correlation between the amount of weight lost and $\Delta$ HOMA-IR $(r=0.24, p<$ 0.0001 ), there was heterogeneity both in the amount of weight lost and the $\triangle$ HOMA-IR over the corresponding 6 month period (Fig. 1). For example, one participant lost $22.2 \mathrm{~kg}$ and had an improvement in HOMA of 6.64, 
Table 1 Baseline clinical characteristics of study population
Values are mean \pm SD unless stated otherwise

\begin{tabular}{|c|c|c|}
\hline Variable & $\begin{array}{l}\text { Overall WLM population } \\
\text { randomised in } \\
\text { phase II }(n=1,032)\end{array}$ & $\begin{array}{l}\text { WLM population randomly } \\
\text { selected for molecular } \\
\text { profiling }(n=500)\end{array}$ \\
\hline Age (years) & $55.6 \pm 8.7$ & $55.9 \pm 8.7$ \\
\hline \multicolumn{3}{|l|}{ Race $(\%)$} \\
\hline White & 62.4 & 62.6 \\
\hline African-American & 37.6 & 37.4 \\
\hline Sex $(\%$ female $)$ & 63.4 & 63.0 \\
\hline Baseline BMI $\left(\mathrm{kg} / \mathrm{m}^{2}\right)$ & $34.1 \pm 4.8$ & $33.9 \pm 4.7$ \\
\hline Baseline weight (kg) & $96.7 \pm 16.6$ & $96.0 \pm 16.1$ \\
\hline Weight at 6 months $(\mathrm{kg})$ & $88.2 \pm 15.8$ & $87.3 \pm 15.3$ \\
\hline Weight loss (kg) & $8.5 \pm 4.2$ & $8.7 \pm 4.3$ \\
\hline \multicolumn{3}{|l|}{ Medications (\%) } \\
\hline Hypertension & 87.0 & 87.6 \\
\hline Lipid & 39.8 & 38.8 \\
\hline \multicolumn{3}{|l|}{ Education $(\%)$} \\
\hline Some college or less & 38.4 & 37.4 \\
\hline College degree & 61.6 & 62.6 \\
\hline Smoking (\%) & 4.3 & 4.0 \\
\hline \multicolumn{3}{|l|}{ Household income/year (\%) } \\
\hline$<$ US\$60,000 or no answer & 42.6 & 42.2 \\
\hline$\geq \mathrm{US} \$ 60,000$ & 57.4 & 57.8 \\
\hline Energy intake at baseline $(\mathrm{kJ} /$ day $)$ & $6681.0 \pm 1929.2$ & $8198.1 \pm 3718.3$ \\
\hline Glycaemic load at baseline & $113.3 \pm 58.0$ & $112.7 \pm 56.7$ \\
\hline
\end{tabular}

whereas another participant who lost a greater amount of weight $(28.2 \mathrm{~kg})$ had only a minimal improvement in HOMA (0.66). A third participant had no change in IR $(\triangle \mathrm{HOMA}=0.06)$ despite $18 \mathrm{~kg}$ of weight lost. In fact, $22 \%$ of study participants had no improvement in HOMA-IR. Similar heterogeneity was observed for per cent weight loss with per cent change in HOMA-IR. These results suggest

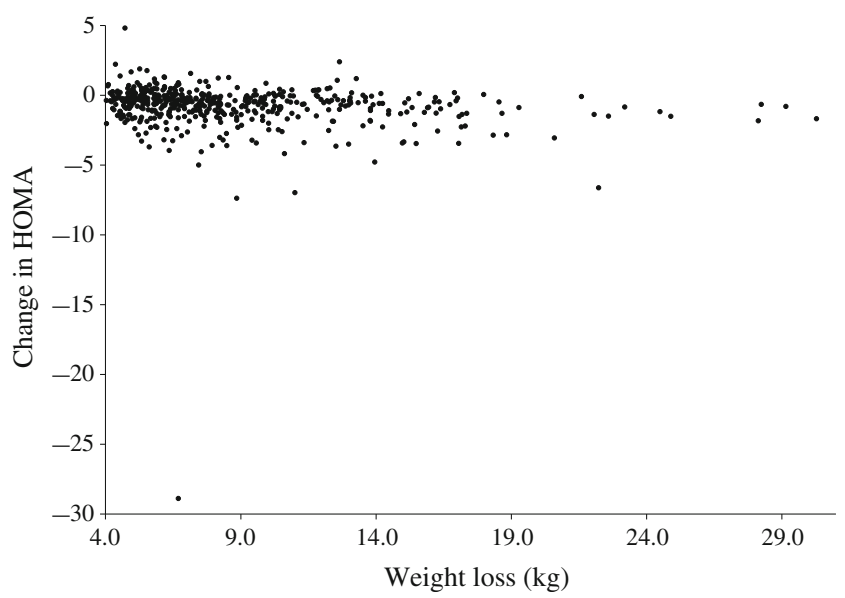

Fig. 1 Scatterplot showing change in weight and $\triangle$ HOMA demonstrating only a modest correlation between these two traits, with marked heterogeneity in $\triangle$ HOMA with varying amounts of weight loss that improvement in IR was neither solely nor primarily related to the amount of weight lost.

\section{Clinical model for $\triangle \mathrm{HOMA}-\mathrm{IR}$}

Several clinical characteristics were associated with $\triangle$ HOMA-IR in univariate analyses (Table 2). However, in multivariable analyses, only sex, education, income, baseline weight, amount of weight lost and the HEI remained independently associated with $\triangle$ HOMA-IR. Therefore, these variables were used for adjustment in subsequent analyses of the relationship between metabolomic factors and $\triangle$ HOMA-IR.

\section{Metabolomic predictors of $\triangle$ HOMA-IR}

PCA identified 13 factors composed of correlated metabolites (Table 3), with metabolites clustered within factors in biologically plausible pathways and consistent with previous findings [7-9, 11, 12]. Two factors were associated with $\triangle$ HOMA-IR in univariate analyses (factor 3 [branched-chain amino acids (BCAAs) and related catabolites] and factor 10 [amino acids], Table 4). In multivariable analyses, only factor 3 remained associated with $\triangle$ HOMAIR. After correction for multiple comparisons at the level of factors, factor 3 remained significant (Bonferroni corrected 
Table 2 Clinical model for $\triangle \mathrm{HOMA}$

\begin{tabular}{|c|c|c|c|c|}
\hline Variable & $F$ value & Univariate $p$ value & Multivariable $p$ value & Greater improvement in IR associated with: \\
\hline $\operatorname{Sex}^{\mathrm{a}}$ & 12.17 & 0.0005 & 0.04 & Men \\
\hline Race & 3.82 & 0.05 & 0.57 & White \\
\hline Age & 0.27 & 0.60 & 0.06 & \\
\hline Smoking & 1.65 & 0.19 & 0.33 & \\
\hline Education $^{\mathrm{a}}$ & 0.88 & 0.45 & 0.02 & Less education \\
\hline Income $^{\mathrm{a}}$ & 0.48 & 0.62 & 0.03 & Lower income \\
\hline Amount of weight lost $\mathrm{t}^{\mathrm{a}}$ & 10.85 & 0.001 & $<0.0001$ & More weight lost \\
\hline Entry weight ${ }^{\mathrm{a}}$ & 18.04 & 0.00003 & $<0.0001$ & Higher entry weight \\
\hline Medications for dyslipidaemia & 7.17 & 0.008 & 0.33 & Not taking dyslipidaemia medications at entry \\
\hline Medications for hypertension & 0.03 & 0.87 & 0.43 & \\
\hline$\%$ Energy from alcohol & 7.21 & 0.007 & 0.75 & Higher alcohol intake at entry \\
\hline $\mathrm{HEI}^{\mathrm{a}}$ & 1.43 & 0.23 & 0.02 & Higher HEI at entry \\
\hline Glycaemic load & 4.46 & 0.04 & 0.42 & Higher glycaemic load at entry \\
\hline Depression score & 0.008 & 0.93 & 0.62 & \\
\hline MVPA & 0.43 & 0.51 & 0.67 & \\
\hline
\end{tabular}

MVPA, moderate to vigorous physical activity

${ }^{a}$ Variables that were significant in the multivariable model and therefore were included as the base clinical model

$p<0.004)$ and therefore we chose to focus on this factor, composed of BCAAs and related metabolites (BCAA factor), for the remainder of the analyses.

Baseline BCAA factor levels were correlated with baseline HOMA-IR $(r=0.50, p<0.0001), 6$ month HOMA-IR $(r=$ $0.28, p<0.0001)$ and $\Delta$ HOMA-IR $(r=-0.38, p<0.0001)$. When baseline BCAA factor levels were divided into quartiles, there was a linear relationship of HOMA-IR measures across BCAA factor quartiles (Table 5), such that the greater the BCAA factor level at baseline, the greater the improvement in IR with weight loss. The clinical model explained $5.5 \%$ of variability in $\triangle \mathrm{HOMA}-\mathrm{IR}$ and the model inclusive of baseline BCAA factor levels and clinical variables explained $10.8 \%$ of the variance in $\triangle \mathrm{HOMA}-\mathrm{IR}$.

In the New York validation cohort, in the combined gastric bypass surgery and matched diet groups, PCA also identified a factor composed of the branched chain and other amino acids (leucine/isoleucine, phenylalanine, tyrosine, alanine, ornithine),

Table 3 PCA: the 13 factors identified from PCA, with an overall description of the factor (name), individual metabolites composing that factor (i.e. those metabolites with an absolute value of the factor load $\geq 0.4$ for that factor), the eigenvalue for the factor and the variance for the factor

\begin{tabular}{|c|c|c|c|c|}
\hline Factor & Name & Individual components & Eigenvalue & Variance \\
\hline 1 & Medium-chain acylcarnitines & $\begin{array}{c}\text { C8, C10:1, C10, C10-OH:C8-DC,C12:1, C12, C14:2, } \\
\text { C14:1, C14, C8:1-DC, C16:2, C16.1 acylcarnitines }\end{array}$ & 14.94 & 0.25 \\
\hline 2 & Medium-chain dicarboxyl-acylcarnitines & $\begin{array}{l}\text { Ci4-DC/C4-DC, C6-DC, C10-OH/C8-DC, C12:1, } \\
\text { C12-OH/C10-DC, C14:1-OH/C12:1-DC, } \\
\text { C14-OH/C12-DC, C8:1-DC }\end{array}$ & 6.21 & 0.11 \\
\hline 3 & BCAA-related & Ala, Pro, Val, Leu/Ile, Met, Phe, Tyr, Glx, Orn & 3.58 & 0.06 \\
\hline 4 & Ketone-related & $\mathrm{C} 2$, C4-OH, C16:1, total ketones, 3-OH butyrate, NEFA & 3.34 & 0.06 \\
\hline 5 & Long-chain dicarboxyl-acylcarnitines & $\begin{array}{l}\mathrm{C} 18: 1-\mathrm{OH} / \mathrm{C} 16: 1-\mathrm{DC}, \mathrm{C} 18-\mathrm{OH} / \mathrm{C} 16-\mathrm{DC}, \mathrm{C} 20 \\
\mathrm{C} 20: 1-\mathrm{OH} / \mathrm{C} 18: 1-\mathrm{DC}, \mathrm{C} 20-\mathrm{OH} / \mathrm{C} 18-\mathrm{DC}\end{array}$ & 2.36 & 0.04 \\
\hline 6 & Medium-chain acylcarnitines & C14, C16, C18:1, C18, C16:1-OH/C14:1-DC & 2.21 & 0.04 \\
\hline 7 & Medium-chain acylcarnitines & $\mathrm{C} 8: 1, \mathrm{C} 10: 3, \mathrm{C} 10: 2$ & 2.05 & 0.03 \\
\hline 8 & Short-chain acylcarnitines & $\mathrm{C} 3, \mathrm{C} 4 / \mathrm{Ci} 4, \mathrm{C} 5 \mathrm{~s}$ & 1.76 & 0.03 \\
\hline 9 & Long-chain acylcarnitines & $\mathrm{C} 18: 2, \mathrm{C} 18: 1, \mathrm{C} 20: 4$ & 1.40 & 0.02 \\
\hline 10 & Amino acids & Gly, Ser, Arg & 1.25 & 0.02 \\
\hline 11 & Urea cycle & Orn, Cit, C5-DC & 1.16 & 0.02 \\
\hline 12 & Miscellaneous & $\mathrm{C} 5: 1$ & 1.10 & 0.02 \\
\hline 13 & Miscellaneous & His $(-)$, Asx & 1.07 & 0.02 \\
\hline
\end{tabular}


Table 4 PCA-derived factors and association with $\triangle \mathrm{HOMA}$

\begin{tabular}{|c|c|c|c|c|c|}
\hline Factor & Description & $F$ value & Unadjusted $p$ value & Adjusted $p$ value ${ }^{\mathrm{a}}$ & Effect size $(95 \% \mathrm{CI})^{\mathrm{b}}$ \\
\hline 1 & Medium-chain acylcarnitines & 0.08 & 0.78 & NS & $-0.022(-0.175,0.131)$ \\
\hline 2 & Medium-chain dicarboxyl-acylcarnitines & 1.96 & 0.16 & NS & $-0.109(-0.262,0.044)$ \\
\hline 3 & BCAA-related & 47.82 & $<0.0001$ & $<0.0001$ & $-0.513(-0.659,-0.367)$ \\
\hline 4 & Ketone-related & 1.19 & 0.28 & NS & $0.085(-0.068,0.237)$ \\
\hline 5 & Long-chain dicarboxyl-acylcarnitines & 0.32 & 0.57 & NS & $-0.044(-0.197,0.109)$ \\
\hline 6 & Medium-chain acylcarnitines & 3.25 & 0.07 & NS & $-0.140(-0.293,0.013)$ \\
\hline 7 & Medium-chain acylcarnitines & 0.19 & 0.66 & NS & $-0.034(-0.187,0.119)$ \\
\hline 8 & Short-chain acylcarnitines & 3.08 & 0.08 & NS & $-0.136(-0.288,0.016)$ \\
\hline 9 & Long-chain acylcarnitines & 2.06 & 0.15 & NS & $0.111(-0.041,0.264)$ \\
\hline 10 & Amino acids & 4.17 & 0.04 & NS & $0.158(0.006,0.310)$ \\
\hline 11 & Urea cycle & 3.03 & 0.08 & NS & $0.135(-0.018,0.288)$ \\
\hline 12 & Miscellaneous & 0.82 & 0.37 & NS & $0.070(-0.082,0.223)$ \\
\hline 13 & Miscellaneous & 0.11 & 0.74 & NS & $0.027(-0.129,0.182)$ \\
\hline
\end{tabular}

${ }^{a}$ Adjusted for sex, education, income, entry weight, change in weight, HEI (Healthy Eating Index)

${ }^{\mathrm{b}}$ Per 1 unit change in factor level

similar to the BCAA factor identified in the WLM cohort. In the validation cohort, the BCAA factor, but not weight loss, also predicted $\triangle$ HOMA-IR ( $p=0.02$ ) for both groups, and after adjusting for group (diet vs gastric bypass surgery) $(p=0.007)$.

Relationship between BCAA intake, BCAA factor levels and IR

BCAAs are found in dietary proteins; hence, differences in baseline peripheral blood BCAA levels (and subsequent improvement in IR with weight loss) could be reflective of differences in dietary BCAA intake. In an exploratory fashion, we therefore examined reported baseline and 6 month intake of dietary BCAA. BCAA intake was defined as a sum of dietary valine, leucine and isoleucine intake and peripheral blood BCAA levels were defined as a sum of peripheral blood valine and leucine/isoleucine levels. BCAA intake was weakly correlated with blood BCAA levels at baseline $(r=0.14$, $p=0.003)$ and at 6 months $(r=0.14, p=0.002)$. Change in BCAA intake over the 6 months was not correlated with change in blood BCAA levels over that time period $(r=0.04$, $p=0.39)$. Baseline BCAA intake was weakly correlated with $\triangle$ HOMA-IR $(r=-0.14, p=0.002)$ and change in BCAA intake was not correlated with $\triangle$ HOMA-IR $(r=-0.01, p=$ 0.82). These data, although exploratory, suggest that dietary BCAA intake accounts only for a small portion of peripheral blood BCAA levels and their change during weight loss.

\section{Change in BCAAs with weight loss}

Our previous work in a small cohort has shown that BCAA levels decrease with weight loss [13]. We found similar results in this larger cohort. Specifically, the BCAAs valine and leucine/isoleucine decreased significantly with weight loss $(p=0.005$ and $p<0.0001$, respectively), as did the aromatic amino acids phenylalanine and tyrosine $(p=0.002$ and $p<0.0001$, respectively).

Table 5 Mean levels of baseline HOMA-IR, 6 month HOMA-IR and $\triangle$ HOMA-IR stratified by quartiles of factor $3^{\mathrm{a}}$, demonstrating a linear relationship of HOMA-IR across quartiles

\begin{tabular}{|c|c|c|c|c|c|}
\hline \multirow[t]{2}{*}{ Variable } & \multicolumn{4}{|c|}{ Baseline factor 3 levels } & \multirow[t]{2}{*}{$p$ value } \\
\hline & Quartile 1 & Quartile 2 & Quartile 3 & Quartile 4 & \\
\hline Baseline HOMA-IR & $1.6 \pm 0.9$ & $2.0 \pm 1.2$ & $2.6 \pm 1.6$ & $3.6 \pm 3.7$ & $<0.0001$ \\
\hline 6-month HOMA-IR & $1.3 \pm 0.8$ & $1.4 \pm 0.8$ & $1.7 \pm 1.1$ & $2.1 \pm 1.5$ & $<0.0001$ \\
\hline$\Delta$ HOMA-IR & $-0.2 \pm 0.7$ & $-0.5 \pm 0.9$ & $-0.9 \pm 1.3$ & $-1.5 \pm 2.9$ & $<0.0001$ \\
\hline
\end{tabular}

Values are mean $\pm \mathrm{SD}$

${ }^{a}$ PCA-derived factor consisting of the BCAAs valine, leucine and isoleucine; the aromatic amino acids phenylalanine and tyrosine; and other amino acids (alanine, proline, methionine, glutamate/glutamine and ornithine) 


\section{Discussion}

In this sub-study of the WLM trial, we have used targeted metabolomic profiling to identify a biosignature reporting on BCAA metabolism that is associated with IR in nondiabetic overweight/obese individuals and, more importantly, predicts improvement in IR with moderate weight loss independent of the amount of weight lost. In fact, in our study, the amount of weight lost was only modestly correlated with the degree of improvement in IR. To our knowledge, this is the first biomarker that has been identified for predicting health benefits in the context of weight loss, thereby helping to dissect the known clinical heterogeneity of these benefits. These results could help identify individuals who would more likely benefit from moderate weight loss and potentially elucidate novel mechanisms of IR in obese individuals.

BCAAs comprise approximately $40 \%$ of the free essential amino acids in blood. They play important roles in maintenance and growth of skeletal muscle, are used as an energy source during exercise, and can serve as gluconeogenic precursors. Increased BCAA catabolism, driven by higher levels of these analytes in the blood, may explain the higher levels of glutamate, alanine and proline that comprise factor 3 (see Fig. 2). Further, the BCAAs share a competitive transport system with the aromatic amino acids, thereby potentially explaining the clustering of phenylalanine and tyrosine in this factor [14], not only in the current study but in several other reports from our group [7-9, 11, 12]. In a previous report, C3 and C5 acylcarnitines, by-products of mitochondrial catabolism of BCAAs, also contributed to a BCAA-related factor in obese humans [7]. In the current report, C3 and C5 acylcarnitines did not quite reach the a priori defined threshold factor load of 0.40 to be included as a contributor to factor 3 , but did have moderately high factor loads $(0.34$ and 0.33 , respectively), further corroborating the analytical and biological clustering of these metabolites in this factor. In fact, C3, C4 and C5 acylcarnitines also grouped as an independent factor (factor 8, short chain acylcarnitines), which nearly reached a significant association with $\triangle$ HOMA-IR (unadjusted $p=0.08$ ).

These results are particularly provocative in light of our previous studies implicating BCAAs in IR. We have shown that a similar cluster of BCAAs and related catabolites distinguishes lean from obese individuals and is associated with IR [7]. These results were validated in an independent cross-sectional study of individuals at risk for type 2 diabetes mellitus undergoing a frequently sampled intravenous glucose tolerance test [11]. In a third study involving application of metabolomics to participants with and without coronary artery disease (CAD), we found that a PCA-derived BCAA factor was associated with CAD [8]. In a fourth study, we found that BCAAs and related metabolites differentiated insulin resistant from insulin sensitive participants in Chinese and Asian-Indian cohorts, each matched for BMI (BMI of 24) [12]. Finally, Shaham and associates have reported that BCAAs are among a cluster of metabolites that change more significantly in insulin resistant vs normal participants during a glucose tolerance test [15].

More recently, we have reported on changes in metabolites occurring in response to bariatric surgery or dietary intervention [13]. We found that the intervention with the
Fig. 2 Schematic of mitochondrial BCAA catabolism, demonstrating key metabolites in this pathway, most of which clustered in factor 3

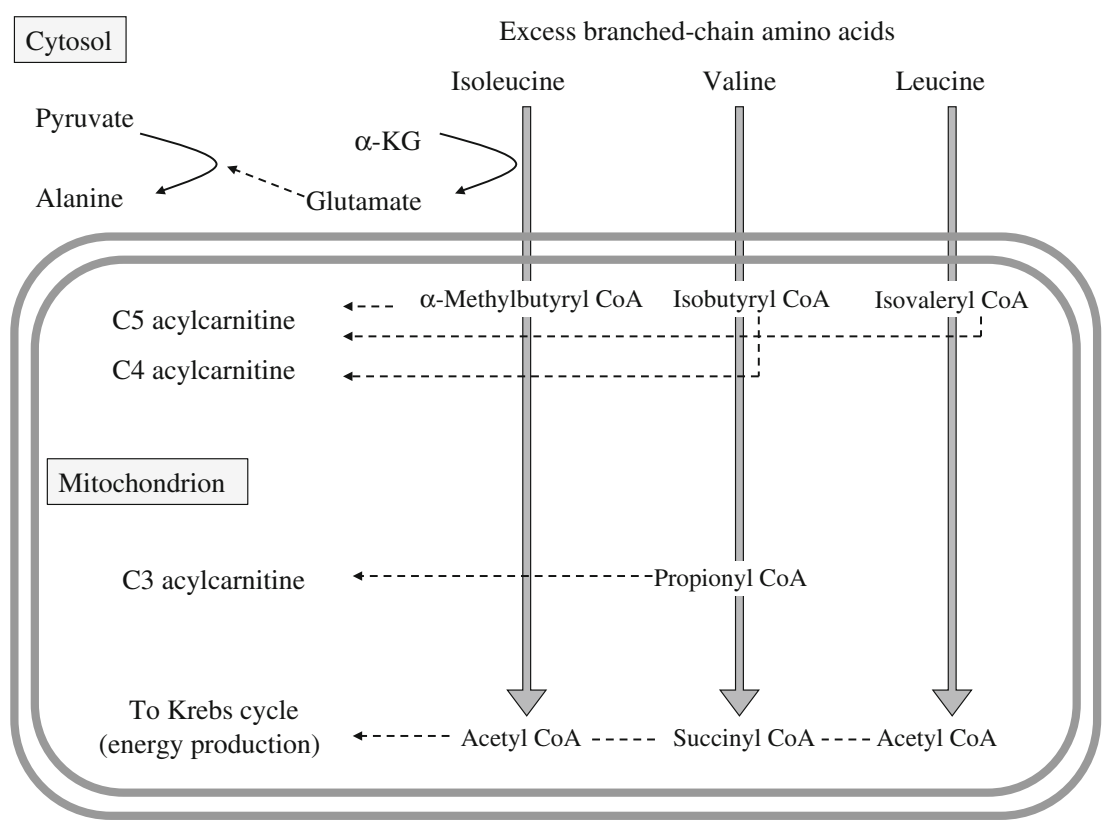


more robust impact on glucose homeostasis, bariatric surgery, also has the stronger impact on lowering circulating levels of BCAAs and related metabolites. Unlike the present study, that observational study did not attempt to use baseline BCAA levels to predict improvement in insulin resistance in response to a dietary weight loss intervention. Another recent observational study found that a cluster of BCAAs and aromatic amino acids predicts development of type 2 diabetes up to 12 years in advance [16]. Finally, another recent study performed in Zucker obese rats treated with four thiazolidinedione (TZD) drugs demonstrates a strong and unique association between the relative efficacy of the drugs for glycaemic control and regulation of a panel of genes encoding enzymes in the BCAA catabolic pathway in adipose tissue [17]. The same group has also demonstrated a strong correlation between BCAA catabolic genes, insulin resistance and TZD action in humans [18]. The current study adds to this exciting new information by demonstrating for the first time that baseline levels of BCAAs and related metabolites can predict the outcome of a therapeutic intervention.

The clinical and biological implications of our results are as follows. While HOMA-IR is a strong marker of insulin resistance, it is not used clinically and remains primarily an epidemiological research tool. As such, in this era of the pursuit of personalised medicine, there is a need to identify novel biomarkers that could help guide intervention strategies in obesity. Our results suggest that BCAA factor levels could help identify those patients who would benefit the most from the moderate weight loss incurred through behavioural interventions, and thus demonstrate for the first time that a blood-borne biomarker could be used for tailoring of such therapies. Importantly, BCAAs and related metabolites were unique among a much larger and more diverse set of metabolites evaluated in predicting change in HOMA-IR, and therefore suggest a potential mechanism for the heterogeneity in health benefits obtained from weight loss.

Our previous work has shown that BCAAs and related metabolites are elevated in insulin resistant vs insulin sensitive participants with an average BMI of $24 \mathrm{~kg} / \mathrm{m}^{2}$, despite equal rates of protein intake [12]. No differences in protein intake were found in the longitudinal study of diabetic vs non-diabetic participants in the Framingham cohort, despite higher levels of BCAAs and aromatic amino acids in the former group [16], and in this study we found that dietary BCAA intake is only weakly correlated with blood BCAA levels. On the other hand, higher BCAA levels were found in obese and insulin resistant participants compared with lean and insulin sensitive participants in our prior case/control study and this difference was associated with a higher rate of protein intake in the former group [7]. We have also shown that supplementation of high-fat diets with BCAAs causes a rise in circulating BCAAs and related metabolites and contributes to IR in rats [7]. Thus, diet remains as a potential contributor to the increase in BCAAs in insulin resistant individuals. However, other factors can clearly contribute; for example, it has recently been shown both in rodent models and humans that obesity-related increases in BCAA levels are associated with reduced activities of key BCAA catabolic enzymes in liver and adipose tissue [19, 20], suggesting one mechanism by which BCAA levels could rise in the blood of obese and insulin resistant participants. Rates of protein turnover could contribute as well, and further studies are required to fully understand changes in flux through contributory pathways leading to a highly replicable signature of elevated BCAAs and related metabolites in insulin resistant states. Differences in BCAA levels could also be due to genetic differences in genes regulating the BCAA catabolic pathway, supported by our work showing that BCAA levels are highly heritable in human families burdened with early-onset cardiovascular disease [9]. Overall, our results suggest that inter-individual differences in the BCAA catabolic pathway may contribute to the heterogeneity of improvement in insulin resistance with weight loss.

This study is the largest to use high-throughput metabolomic profiling to understand health benefits occurring as a result of weight loss. A large, well-characterised clinical trial population with serial blood samples and food intake data, in combination with application of targeted metabolomic profiling, facilitated our identification of this biosignature, and has given us insights into possible mechanisms of disease pathology. The WLM population consisted of non-diabetic individuals, and hence these results have implications for understanding IR in the absence of overt type 2 diabetes mellitus. Importantly, the signature also predicts improvements in insulin sensitivity in response to diet- and gastric bypass-induced weight loss in participants with type 2 diabetes mellitus, as shown in the New York validation cohort.

Our study does have limitations. All participants from the primary cohort underwent behavioural intervention for weight loss, and thus, we cannot make definitive conclusions about the generalisability of these results to other weight loss interventions. However, our results were confirmed in a small validation cohort of bariatric surgery and caloric restriction participants. Baseline factor 3 levels were strongly associated with $\triangle$ HOMA-IR in a multivariable model. However, given the strong co-linearity of baseline factor 3 levels with baseline HOMA-IR $(r=0.50, p<0.0001)$, consistent with our previous work showing that BCAA levels are highly correlated with IR, the independent relationship between baseline factor 3 levels and $\triangle$ HOMA-IR is attenuated, although still significant, in a model adjusted for amount of weight lost and 
baseline HOMA $(p=0.01)$. This relationship is attenuated further in the full model inclusive of clinical variables and baseline HOMA $(p=0.07)$, which is most likely due to a decrease in power because of a loss of nine individuals missing data for one of the clinical variables. Regardless, this biosignature serves as a unique biomarker capable of identifying individuals incurring the greatest health benefits from moderate weight loss even after accounting for routinely collected clinical variables, and elucidates a biological mechanism for this heterogeneity of effect.

Although HOMA-IR is a commonly used epidemiological tool for assessment of IR and $\beta$-cell function, and has been shown to correlate with more complicated and costly 'gold standard' measures of IR (i.e. euglycaemic-hyperinsulinaemic clamp), it may not perform as well in certain subgroups [21]. Medications could influence metabolite levels and confound the relationship between the BCAA levels and prediction of improvement in insulin resistance. However, we found no difference in the use of dyslipidaemia or anti-hypertensive medications within the BCAA factor quartiles outlined in Table 5 ( $p=0.33$ for medications for dyslipidaemia, $p=0.37$ for statins, $p=0.73$ for anti-hypertension medications). Finally, the full model including clinical characteristics and BCAA factor levels only explains $10.8 \%$ of variance in $\triangle$ HOMA-IR; thus, other yet to be defined variables must contribute to the heterogeneity of changes in IR occurring with moderate weight loss. In addition, it is important to note that we are not recommending the use of BCAA markers in clinical practice at this time. The ultimate clinical utility of this biomarker will depend on further validation and deeper understanding of its pathophysiological and predictive roles.

In conclusion, a metabolite signature representing BCAA catabolism is correlated with IR and predicts improvement in IR with moderate weight loss, independent of the amount of weight lost. These results may help identify individuals most likely to benefit from moderate weight loss and elucidate novel mechanisms of IR in obesity.

\footnotetext{
Acknowledgements We are grateful to all participants in the WLM clinical trial, and to G. Meltesen and A. Bauck at the WLM Coordinating Center, Center for Health Research, Portland, OR, USA. This study was supported by UO1 grants HL68734, HL68676, HL68790, HL68920, and HL68955. It was also supported by funding from the Measurement to Understand Re-Classification of Disease of Cabarrus and Kannapolis (MURDOCK) Study and by Grant Number 1UL1 RR024128-01 from the National Center for Research Resources (NCRR), a component of the National Institutes of Health (NIH) and NIH Roadmap for Medical Research, and its contents are solely the responsibility of the authors and do not necessarily represent the official view of NCRR or NIH. Information on NCRR is available at www.ncrr.nih.gov/. Information on Re-engineering the Clinical Research Enterprise can be obtained from http://nihroadmap.nih.gov/clinicalresearch/overviewl-translational. asp. The New York Obesity Research Center validation cohort was funded by grants from the American Diabetes Association CR-7-05 CR18, NIH R01-DK67561, GCRC 1 UL1 RR024156-02, ORC DK26687, DERC DK-63068-05, NIMH grant MH-48858.
}

Contribution statement SHS had full access to all the data and takes responsibility for the integrity of the data and accuracy of the data analysis. SHS, DRC, CSH, SN, CBT, BL, LFL, PJB, VJS, JFH, LJA, BB, CBN and LPS contributed to study concept and design. SHS, DRC, CSH, SN, CBT, RDS, MJM, BRW, JRB, BL, PG, JT, LFL, BB, CBN and LPS contributed to analysis and interpretation of data. SHS, CBN and LPS contributed to drafting of the manuscript. SHS, DRC, CSH, SN, CBT, RDS, MJM, BRW, JRB, BL, PG, JT, PJB, VJS, JFH, LJA, LFL, BB, CBN and LPS contributed to critical revision of the manuscript for intellectual content. SHS, DRC and $\mathrm{CSH}$ contributed to statistical analysis. All the authors gave approval of the final version to be published.

Duality of interest The authors declare that there is no duality of interest associated with this manuscript.

\section{References}

1. Knowler WC, Barrett-Connor E, Fowler SE et al (2002) Reduction in the incidence of type 2 diabetes with lifestyle intervention or metformin. N Engl J Med 346:393-403

2. Tuomilehto J, Lindstrom J, Eriksson JG et al (2001) Prevention of type 2 diabetes mellitus by changes in lifestyle among subjects with impaired glucose tolerance. N Engl J Med 344:1343-1350

3. Svetkey LP, Stevens VJ, Brantley PJ et al (2008) Comparison of strategies for sustaining weight loss: the weight loss maintenance randomized controlled trial. JAMA 299:1139-1148

4. Harlan LC, Block G (1990) Use of adjustment factors with a brief food frequency questionnaire to obtain nutrient values. Epidemiology $1: 224-231$

5. McCullough ML, Feskanich D, Stampfer MJ et al (2002) Diet quality and major chronic disease risk in men and women: moving toward improved dietary guidance. Am J Clin Nutr 76:1261-1271

6. Laferrere B, Teixeira J, McGinty J et al (2008) Effect of weight loss by gastric bypass surgery versus hypocaloric diet on glucose and incretin levels in patients with type 2 diabetes. J Clin Endocrinol Metab 93:2479-2485

7. Newgard CB, An J, Bain JR et al (2009) A branched-chain amino acid-related metabolic signature that differentiates obese and lean humans and contributes to insulin resistance. Cell Metab 9:311-326

8. Shah SH, Bain JR, Muehlbauer MJ et al (2010) Association of a peripheral blood metabolic profile with coronary artery disease and risk of subsequent cardiovascular events. Circ Cardiovasc Genet 3:207-214

9. Shah SH, Hauser ER, Bain JR et al (2009) High heritability of metabolomic profiles in families burdened with premature cardiovascular disease. Mol Syst Biol 5:258

10. Matthews DR, Hosker JP, Rudenski AS, Naylor BA, Treacher DF, Turner RC (1985) Homeostasis model assessment: insulin resistance and beta-cell function from fasting plasma glucose and insulin concentrations in man. Diabetologia 28:412-419

11. Huffman KM, Shah SH, Stevens RD et al (2009) Relationships between circulating metabolic intermediates and insulin action in overweight to obese, inactive men and women. Diabetes Care 32:1678-1683

12. Tai ES, Tan ML, Stevens RD et al (2010) Insulin resistance is associated with a metabolic profile of altered protein metabolism in Chinese and Asian-Indian men. Diabetologia 53:757-767

13. Laferrere B, Reilly D, Arias S et al (2011) Differential metabolic impact of gastric bypass surgery versus dietary intervention in obese diabetic subjects despite identical weight loss. Sci Transl Med 3:80re82 
14. Fernstrom JD (2005) Branched-chain amino acids and brain function. J Nutr 135:1539S-1546S

15. Shaham O, Wei R, Wang TJ et al (2008) Metabolic profiling of the human response to a glucose challenge reveals distinct axes of insulin sensitivity. Mol Syst Biol 4:214

16. Wang TJ, Larson MG, Vasan RS et al (2011) Metabolite profiles and the risk of developing diabetes. Nat Med 17:448-453

17. Hsiao G, Chapman J, Ofrecio JM et al (2011) Multi-tissue, selective PPARgamma modulation of insulin sensitivity and metabolic pathways in obese rats. Am J Physiol Endocrinol Metab 300:E164-E174

18. Sears DD, Hsiao G, Hsiao A et al (2009) Mechanisms of human insulin resistance and thiazolidinedione-mediated insulin sensitization. Proc Natl Acad Sci USA 106:18745-18750
19. Herman MA, She P, Peroni OD, Lynch CJ, Kahn BB (2010) Adipose tissue branched chain amino acid (BCAA) metabolism modulates circulating BCAA levels. J Biol Chem 285:1134811356

20. She P, van Horn C, Reid T, Hutson SM, Cooney RN, Lynch CJ (2007) Obesity-related elevations in plasma leucine are associated with alterations in enzymes involved in branched-chain amino acid metabolism. Am J Physiol Endocrinol Metab 293:E1552E1563

21. Anderson RL, Hamman RF, Savage PJ et al (1995) Exploration of simple insulin sensitivity measures derived from frequently sampled intravenous glucose tolerance (FSIGT) tests. The Insulin Resistance Atherosclerosis Study. Am J Epidemiol 142:724-732 